\title{
œcreative
}

\section{The automorphism groups of non-edge-transitive rose window graphs}

\author{
Edward Dobson * \\ Department of Mathematics and Statistics, Mississippi State University \\ PO Drawer MA Mississippi State, MS 39762 \\ István Kovács , Štefko Miklavič \\ UP IAM and UP FAMNIT, University of Primorska \\ Muzejski trg 2, 6000 Koper, Slovenia
}

Received 2 August 2012, accepted 7 September 2012, published online 20 June 2014

\begin{abstract}
In this paper, we will determine the full automorphism groups of rose window graphs that are not edge-transitive. As the full automorphism groups of edge-transitive rose window graphs have been determined, this will complete the problem of calculating the full automorphism group of rose window graphs. As a corollary, we determine which rose window graphs are vertex-transitive. Finally, we determine the isomorphism classes of non-edge-transitive rose window graphs.
\end{abstract}

Keywords: Rose window graphs, automorphism group, isomorphism problem, vertex-transitive graph. Math. Subj. Class.: 05E18

\section{Introduction}

Rose windows graphs are defined as follows (we are using the notation and terminology as in [18]).

Definition 1.1. Let $n$ be a positive integer and $a, r \in \mathbb{Z}_{n}$ (so arithmetic with $a$ and $r$ is done modulo $n$ ). The rose window graph $R_{n}(a, r)$ is defined to be the graph with vertex set $V=\left\{A_{i}, B_{i}: i \in \mathbb{Z}_{n}\right\}$ and four kinds of edges:

- $A_{i} A_{i+1}$ These edges are called rim edges.

\footnotetext{
*Project sponsored by the National Security Agency under Grant Number H98230-11-1-0179.

E-mail address: dobson@math.msstate.edu (Edward Dobson), istvan.kovacs@upr.si (István Kovács), stefko.miklavic@upr.si (Štefko Miklavič)
} 
- $A_{i} B_{i}$ These edges are called in-spoke edges.

- $A_{i+a} B_{i}$ These edges are called out-spoke edges.

- $B_{i} B_{i+r}$ These edges are called hub edges.

Rose window graphs were introduced recently by Steve Wilson [18], whose initial motivation was concerned with determining which of these graphs are edge-transitive (and if so what is their full automorphism group), as well as which of these graphs are the underlying graph of a rotary map. He proposed four conjectures concerning questions that he was interested in, and subsequently all of the conjectures have been shown to be true. Edge-transitive rose window graphs were characterized in [5, Theorem 1.2], verifying [18, Conjecture 11] (a graph is edge-transitive if its automorphism group acts transitively on the set of edges). The full automorphism groups of edge-transitive rose window graphs was determined in [6, $\S 3]$, verifying [18, Conjectures 3 and 5]. The rose window graphs which are the underlying graph of a rotary map were found in [6, Theorem 1.1], answering [18, Question 3], Finally, [18, Conjecture 6] suggesting certain rose window graphs are isomorphic was verified in [6, Theorem 3.6].

Our goal is to essentially complete the work that has already been done regarding calculating the full automorphism groups of rose window graphs, as well determining exactly when two rose window graphs are isomorphic. In this paper, we will calculate the full automorphism groups of rose window graphs that are not edge-transitive (which will finish the problem of determining the full automorphism groups of rose window graphs), see Corollary 3.5 and Corollary 3.9. In Section 4, we will determine the isomorphism classes of rose window graphs that are not edge-transitive. The conclusion of the isomorphism problem for rose window graphs will be given in a sequel to this paper, where the isomorphism classes of edge-transitive rose window graphs will be found.

There are a few additional results in this paper that should be mentioned. First, in Lemma 2.2, we correct a small error in [18, Lemma 2] giving conditions on when a rose window graph has an automorphism that maps every rim edge to a hub edge and vice versa. Also, once the full automorphism groups of rose windows graphs are known, it is relatively straightforward to determine which of these graphs are vertex-transitive, and this is given in Theorem 3.10.

We should point out that our goal is a classical one. Namely, with graphs that have a large amount of symmetry, it is quite standard to ask for their full automorphism groups as well as their isomorphism classes. Perhaps the first family for which this has been done are the generalized Petersen graphs. The automorphism groups of these graphs were obtained by Frucht, Graver, and Watkins [4] in 1971, while the isomorphism classes were found by by Boben, Pisanski, and Žitnik [1]. Using very differrent techniques, Steimle and Staton [16] also determined the isomorphism classes for some, but not all, generalized Petersen graphs, and then used that result to enumerate the generalized Petersen graphs whose isomorphism classes they determined. Using Boben, Pisanski, and Žitnik's determination of the isomorphism classes of all generalized Petersen graphs, Petkovšek and Zakrajšek [9] enumerated generalized Petersen graphs. Determining the isomorphism classes of the rose window graphs should also yield an enumeration of the rose window graphs using techniques similar to those in [9].

Finally, in the last decade or so there has been considerable interest in tetravalent graphs satisfying various properties or in studying certain families of such graphs (for a sample of such work see $[3,7,10,11,12,13,17])$. See also [14], where a census of all locally 
imprimitive tetravalent arc-transitive graphs on up to 640 vertices was computed. This work will certainly contribute to the understanding of such graphs.

\section{Preliminary results}

We first give some obvious automorphisms of rose window graphs. Let $R_{n}(a, r)$ be a fixed rose window graph and let $G$ be the automorphism group of $R_{n}(a, r)$. Observe that

$$
R_{n}(a, r)=R_{n}(a,-r)
$$

Define $\rho, \mu: V \mapsto V$ by

$$
\begin{gathered}
\rho\left(A_{i}\right)=A_{i+1} \quad \text { and } \quad \rho\left(B_{i}\right)=B_{i+1} \quad\left(i \in \mathbb{Z}_{n}\right), \\
\mu\left(A_{i}\right)=A_{-i} \quad \text { and } \quad \mu\left(B_{i}\right)=B_{-a-i} \quad\left(i \in \mathbb{Z}_{n}\right) .
\end{gathered}
$$

Note that $\rho, \mu \in G$, and therefore $\langle\rho, \mu\rangle \leq G$. The action of $\langle\rho, \mu\rangle$ on the set of edges of $R_{n}(a, r)$ has three orbits: the set of rim edges, the set of hub edges and the set of spoke edges.

The following result characterizes edge-transitive rose window graphs in terms of rim and spoke edges (we remark that the full automorphism groups of edge-transitive rose window graphs are given in [5], but the following formulation is nonetheless useful).

Lemma 2.1. The following are equivalent:

(i) $R_{n}(a, r)$ is edge-transitive.

(ii) There is an automorphism of $R_{n}(a, r)$ which sends a rim edge to a spoke edge.

(iii) There is an automorphism of $R_{n}(a, r)$ which sends a spoke edge to a hub edge.

Proof. It is clear that (i) implies (ii). To show that (ii) implies (iii), suppose that $A_{i} A_{i+1}$ is a rim edge mapped to a spoke edge by, say, $\sigma \in G$. Then $\sigma\left(A_{i} A_{i+1}\right)=A_{j} B_{k}$ for some $j, k \in \mathbb{Z}_{n}$, and $\sigma\left(A_{\ell}\right)=B_{k}$ for $\ell=i$ or $i+1$. Of course, $e_{1}=A_{\ell} B_{\ell}$ and $e_{2}=A_{\ell} B_{\ell-a}$ are spoke edges, and $\sigma\left(e_{1}\right)$ and $\sigma\left(e_{2}\right)$ are two edges incident with the spoke edge $A_{j} B_{k}$, and all three of these edges are incident with $\sigma\left(A_{\ell}\right)=B_{k}$. However, $B_{k}$ is incident with two hub edges and two spoke edges, so at least one of $\sigma\left(e_{1}\right)$ and $\sigma\left(e_{2}\right)$ must be a hub edge.

To show (iii) implies (i), recall that the hub, spoke and rim edges are the edge orbits of $\langle\rho, \mu\rangle$. If $\sigma$ maps some spoke edge to a hub edge, we have that $H=\langle\rho, \mu, \sigma\rangle$ has at most two edge orbits, and if there are two edge orbits, these consist of spoke and hub edges in one orbit and rim edges being the other orbit. However, if the rim edges form an orbit, then $H$ must map $\left\{A_{i}: i \in \mathbb{Z}_{n}\right\}$ to itself, and so must map $\left\{B_{i}: i \in \mathbb{Z}_{n}\right\}$ to itself, and so must map hub edges to themselves. This then implies that $H$ has three edge orbits, a contradiction. So $H$ has one edge orbit and $R_{n}(r, a)$ is edge-transitive.

It follows that if $R_{n}(a, r)$ is not edge-transitive, then it has either two orbits or three orbits on edges. If $R_{n}(a, r)$ has two orbits on edges, then one orbit consists of rim and hub edges, and the other consists of spoke edges. If $R_{n}(a, r)$ has three orbits on edges, then the first one consists of rim edges, the second one consists of hub edges, and the third one consists of spoke edges.

Lemma 2 in [18] states that there is an automorphism of $R_{n}(a, r)$ sending rim edges to hub edges and vice-versa if and only if $r^{2} \equiv \pm 1(\bmod n)$ and $r a \equiv \pm a(\bmod n)$. 
However, this is not entirely true. Namely, one can check that the rose window graph $R_{16}(8,3)$ has an automorphism sending rim edges to hub edges and vice-versa via the map $(i, j) \rightarrow(i, 11 j)$. However it is clear that $r^{2}=9 \not \equiv \pm 1(\bmod 16)$. We now wish to give a correct statement of [18, Lemma 2], and begin with a preliminary lemma.

Lemma 2.2. Let $\sigma$ be the automorphism of $R_{n}(a, r)$, which sends every rim edge to a hub edge and vice versa. Assume also that $\sigma\left(A_{0}\right)=B_{0}$ and $\sigma\left(B_{0}\right)=A_{0}$. Then one of the following holds for every $i \in \mathbb{Z}_{n}$ :

(i) $\sigma\left(A_{i}\right)=B_{r i}$ and $\sigma\left(B_{i}\right)=A_{r i}$;

(ii) $\sigma\left(A_{i}\right)=B_{r i}$ and $\sigma\left(B_{i}\right)=A_{(r+a) i}$;

(iii) $\sigma\left(A_{i}\right)=B_{-r i}$ and $\sigma\left(B_{i}\right)=A_{-r i}$;

(iv) $\sigma\left(A_{i}\right)=B_{-r i}$ and $\sigma\left(B_{i}\right)=A_{(-r+a) i}$

Proof. Since $\sigma\left(A_{0}\right)=B_{0}$ and $\sigma\left(A_{1}\right)$ are adjacent, we have $\sigma\left(A_{1}\right) \in\left\{B_{r}, B_{-r}\right\}$. It is easy to see that if $\sigma\left(A_{1}\right)=B_{r}$ then $\sigma\left(A_{i}\right)=B_{r i}$ for $i \in \mathbb{Z}_{n}$, and that if $\sigma\left(A_{1}\right)=B_{-r}$ then $\sigma\left(A_{i}\right)=B_{-r i}$ for $i \in \mathbb{Z}_{n}$. Now let $s \in \mathbb{Z}_{n}$ be such that $\sigma\left(B_{1}\right)=A_{s}$ and note that $\sigma\left(B_{i}\right)=A_{s i}$ for $i \in \mathbb{Z}_{n}$. Moreover, $\sigma\left(A_{1}\right)$ and $\sigma\left(B_{1}\right)=A_{s}$ are adjacent. Therefore, if $\sigma\left(A_{1}\right)=B_{r}$, then $s \in\{r, r+a\}$, and if $\sigma\left(A_{1}\right)=B_{-r}$, then $s \in\{-r,-r+a\}$. The result follows.

Theorem 2.3. Let $n \geq 3$ be an integer and $a, r \in \mathbb{Z}_{n} \backslash\{0\}$. Then there is an automorphism of $R_{n}(a, r)$ sending every rim edge to a hub edge and vice-versa if and only if one of the following holds:

(i) $a \neq n / 2, r^{2} \equiv 1(\bmod n)$ and $r a \equiv \pm a(\bmod n)$;

(ii) $a=n / 2, r^{2} \equiv \pm 1(\bmod n)$ and $r a \equiv \pm a(\bmod n)$;

(iii) $n$ is divisible by $4, \operatorname{gcd}(n, r)=1, a=n / 2$ and $\left(r^{2}+n / 2\right) \equiv \pm 1(\bmod n)$.

Proof. We first show that if (i), (ii) or (iii) holds, then there is an automorphism of $R_{n}(a, r)$ sending rim edges to hub edges and vice-versa. By (2.1) we can assume that $r a \equiv$ $-a(\bmod n)$. Observe that if one of conditions (i), (ii) or (iii) holds, then $\operatorname{gcd}(n, r)=1$. If condition (i) or (ii) holds, then let $\sigma: V \rightarrow V$ be defined by $\sigma\left(A_{i}\right)=B_{r i}$ and $\sigma\left(B_{i}\right)=A_{r i}$. As $\operatorname{gcd}(n, r)=1, \sigma$ is a bijection. It is straightforward to check that $\sigma$ is also an automorphism of $R_{n}(a, r)$.

If condition (iii) holds, then let $\sigma: V \rightarrow V$ be defined by $\sigma\left(A_{i}\right)=B_{r i}$ and $\sigma\left(B_{i}\right)=$ $A_{r i+(n / 2) i}$. Let us show that $\sigma$ is a bijection. As $\operatorname{gcd}(n, r)=1, \sigma$ maps $\left\{A_{i} \mid i \in \mathbb{Z}_{n}\right\}$ to $\left\{B_{i} \mid i \in \mathbb{Z}_{n}\right\}$ bijectively. As $\operatorname{gcd}(n, r)=1, r$ is odd and $n / 2$ is even, $\sigma$ maps $\left\{B_{i} \mid\right.$ $\left.i \in \mathbb{Z}_{n}\right\}$ to $\left\{A_{i} \mid i \in \mathbb{Z}_{n}\right\}$ bijectively. Hence $\sigma$ is a bijection. It is then straightforward to check that $\sigma$ is also an automorphism of $R_{n}(a, r)$.

We now show that if there is an automorphism $\sigma$ of $R_{n}(a, r)$ sending rim edges to hub edges and vice-versa, then either (i), (ii) or (iii) holds. Note that in this case it must be the case that $\operatorname{gcd}(n, r)=1$. Since $\langle\rho, \mu\rangle$ acts transitively on the sets of hub, rim and spoke edges, we may assume (by replacing $\sigma$ by an appropriate element of $\langle\rho, \mu, \sigma\rangle$ ) that $\sigma\left(A_{0}\right)=B_{0}$ and $\sigma\left(B_{0}\right)=A_{0}$. Using (2.1) we can further assume that $\sigma\left(A_{1}\right)=B_{r}$. Therefore, by Lemma 2.2, $\sigma\left(A_{i}\right)=B_{r i}$ and $\sigma\left(B_{i}\right)=A_{s i}$ for $i \in \mathbb{Z}_{n}$, where $s \in\{r, r+a\}$. Since $\sigma^{2}$ sends $A_{0}$ to $A_{0}$ and $A_{1}$ to $A_{r s}$, we have $r s \equiv \pm 1(\bmod n)$. 
Consider an in-spoke $A_{i} B_{i}$ and an out-spoke $B_{i} A_{i+a}$. The automorphism $\sigma$ maps the in-spoke $A_{i} B_{i}$ to $B_{r i} A_{s i}$, and the out-spoke $B_{i} A_{i+a}$ to $A_{s i} B_{r i+r a}$. Hence one of $B_{r i} A_{s i}$ and $A_{s i} B_{r i+r a}$ is an in-spoke, and the other one is an out-spoke. Therefore, for every $i \in \mathbb{Z}_{n}$ either

$$
r i \equiv s i(\bmod n) \quad \text { and } \quad r i+r a+a \equiv s i(\bmod n)
$$

or

$$
r i+r a \equiv s i(\bmod n) \text { and } r i+a \equiv s i(\bmod n) .
$$

Note that if (2.5) holds for $i=0$, then $a=0$, a contradiction. Therefore (2.4) holds for $i=0$, implying $r a \equiv-a(\bmod n)$.

If (2.4) holds for $i=1$, then we have $r=s$. Since $r s \equiv \pm 1(\bmod n)$ this implies $r^{2} \equiv$ $\pm 1(\bmod n)$. If $a=n / 2$, then (ii) holds. If $a \neq n / 2$, then multiplying the congruence $r a \equiv-a(\bmod n)$ by $r$, we obtain $r^{2} a \equiv-a r \equiv a(\bmod n)$. If $r^{2} \equiv-1(\bmod n)$, then $-a \equiv a(\bmod n)$. This implies that $a=n / 2$, a contradiction. So if $a \neq n / 2$, then $r^{2} \equiv 1(\bmod n)$. Thus (i) holds.

Suppose now that $(2.5)$ holds for $i=1$. Then $r+r a \equiv s(\bmod n)$ and $r+a \equiv$ $s(\bmod n)$. The two congruences then imply that $r a \equiv a(\bmod n)$ and $r+a \equiv s(\bmod n)$. Since also $r a \equiv-a(\bmod n)$, we have that $a=n / 2$ and $r$ is odd. Combining together $r+n / 2 \equiv s(\bmod n)$ and $r s \equiv \pm 1(\bmod n)$ gives us $\left(r^{2}+n / 2\right) \equiv \pm 1(\bmod n)$.

Observe that $\sigma\left(B_{n / 2}\right)=A_{s(n / 2)}=A_{(r+n / 2)(n / 2)}$. Suppose $n$ is not divisible by 4 . As $r$ and $n / 2$ are both odd in this case, we have $\sigma\left(B_{n / 2}\right)=A_{0}=\sigma\left(B_{0}\right)$. But this implies that $\sigma$ is not a bijection, a contradiction. Therefore, condition (iii) holds.

It follows from Theorem 2.3 that $R_{n}(a, r)$ has two orbits of edges if and only if one of conditions (i) or (ii) in Theorem 2.3 is satisfied. We will also use the following result.

Lemma 2.4. Assume that $R_{n}(a, r)$ is not edge-transitive and $a=n / 2$. Then at least one of

(i) $r^{2} \equiv \pm 1(\bmod n)$

(ii) $r^{2}+n / 2 \equiv \pm 1(\bmod n)$

does not hold.

Proof. If both (i) and (ii) above hold, then $n / 2$ is congruent to 2,0 or -2 modulo $n$. But this is only possible if $n=4$. If $n=4$, then $r \in\{1,3\}$. In both cases $R_{n}(a, r)$ is edge transitive, a contradiction.

\section{Groups of non edge-transitive rose window graphs}

Before proceeding, we will require some additional notation. Let $N=\operatorname{gcd}(n, r)$ denote the number of inner cycles, and let $L=n / N$ denote the length of an inner cycle. Here an inner cycle is a cycle induced by some set of vertices $\left\{B_{i} \mid i \in \mathbb{Z}_{n}\right\}$. We now define three types of permutations on $V\left(R_{n}(r, a)\right)$. To do this we assume that $n$ is even. For $0 \leq \ell \leq n / 2-1$, we define a permutation on $V\left(R_{n}(r, a)\right)$ by

$$
\alpha_{\ell}=\left(B_{\ell}, B_{\ell+n / 2}\right) .
$$

If $L$ is even, then for $0 \leq \ell \leq N-1$ we let 


$$
\beta_{\ell}=\left(B_{\ell}, B_{\ell+n / 2}\right)\left(B_{\ell+N}, B_{\ell+N+n / 2}\right)\left(B_{\ell+2 N}, B_{\ell+2 N+n / 2}\right) \cdots\left(B_{\ell+n / 2-N}, B_{\ell+n-N}\right) .
$$

Observe that $\beta_{\ell}$ interchanges every two antipodal vertices of the inner cycle containing $B_{\ell}$. If $L$ is odd, then for $0 \leq \ell \leq N / 2-1$ we let

$$
\begin{array}{r}
\gamma_{\ell}=\left(B_{\ell+0}, B_{\ell+n / 2}\right)\left(B_{\ell+N}, B_{\ell+N+n / 2}\right)\left(B_{\ell+2 N}, B_{\ell+2 N+n / 2}\right) \cdots \\
\quad\left(B_{\ell+n-N}, B_{\ell+n-N+n / 2}\right) .
\end{array}
$$

Observe that $\gamma_{\ell}$ interchanges the inner cycle containing $B_{\ell}$ and the inner cycle containing $B_{\ell+n / 2}$.

Lemma 3.1. Assume $n$ is even. Then the following hold:

(i) For $0 \leq \ell \leq n / 2-1$ we have $\alpha_{\ell}=\rho^{\ell} \alpha_{0} \rho^{-\ell}$.

(ii) If $L$ is even, then for $0 \leq \ell \leq N-1$ we have $\beta_{\ell}=\rho^{\ell} \beta_{0} \rho^{-\ell}$.

(iii) If $L$ is odd, then for $0 \leq \ell \leq N / 2-1$ we have $\gamma_{\ell}=\rho^{\ell} \gamma_{0} \rho^{-\ell}$.

Proof. (i) It is straightforward to check that $\left(\rho^{\ell} \alpha_{0} \rho^{-\ell}\right)\left(A_{i}\right)=A_{i}$ for every $i \in \mathbb{Z}_{n}$ and that $\left(\rho^{\ell} \alpha_{0} \rho^{-\ell}\right)\left(B_{i}\right)=B_{i}$ for every $i \in \mathbb{Z}_{n} \backslash\{\ell, \ell+n / 2\}$. Similarly we find that $\rho^{\ell} \alpha_{0} \rho^{-\ell}$ interchanges $B_{\ell}$ and $B_{\ell+n / 2}$. The result follows.

(ii) Since $\beta_{0}=\alpha_{0} \alpha_{N} \alpha_{2 N} \cdots \alpha_{n / 2-N}$ and $\beta_{\ell}=\alpha_{\ell} \alpha_{\ell+N} \alpha_{\ell+2 N} \cdots \alpha_{\ell+n / 2-N}$ the result follows from (i) above.

(iii) Similarly as (ii) above.

Lemma 3.2. Assume $n$ is even. Then the following hold:

(i) If $L=4$ then $\alpha_{\ell}$ is an automorphism of $R_{n}(n / 2, r)$ for $0 \leq \ell \leq n / 2-1$.

(ii) If $L$ is even, $L \neq 4$, then $\beta_{\ell}$ is an automorphism of $R_{n}(n / 2, r)$ for $0 \leq \ell \leq N-1$.

(iii) If $L$ is odd then $\gamma_{\ell}$ is an automorphism of $R_{n}(n / 2, r)$ for $0 \leq \ell \leq N / 2-1$.

Proof. Straightforward.

Lemma 3.3. Let $G_{\mathcal{A}}$ be the point-wise stabiliser of $\left\{A_{0}, A_{1}, \ldots, A_{n-1}\right\}$ in $G$. Then the following hold:

(i) If $a \neq n / 2$ then $G_{\mathcal{A}}$ is trivial.

(ii) If $a=n / 2$ and $L=4$, then $G_{\mathcal{A}}=\left\langle\alpha_{0}, \alpha_{1}, \ldots, \alpha_{n / 2-1}\right\rangle$.

(iii) If $a=n / 2, L$ is even and $L \neq 4$, then $G_{\mathcal{A}}=\left\langle\beta_{0}, \beta_{1}, \ldots, \beta_{N-1}\right\rangle$.

(iv) If $a=n / 2$ and $L$ is odd, then $G_{\mathcal{A}}=\left\langle\gamma_{0}, \gamma_{1}, \ldots, \gamma_{N / 2-1}\right\rangle$.

Proof. Let $\sigma \in G_{\mathcal{A}}$. Since the outer cycle (that is, the $n$-cycle induced by the vertices $\left\{A_{i} \mid\right.$ $\left.\left.i \in \mathbb{Z}_{n}\right\}\right)$ is fixed by $\sigma$, for every $i \in \mathbb{Z}_{n}$ we have either $\sigma\left(B_{i}\right)=B_{i}$ and $\sigma\left(B_{i-a}\right)=B_{i-a}$, or $\sigma\left(B_{i}\right)=B_{i-a}$ and $\sigma\left(B_{i-a}\right)=B_{i}$. If $\sigma$ is nontrivial, then there exists $j \in \mathbb{Z}_{n}$ such that $\sigma\left(B_{j}\right)=B_{j-a}$ and $\sigma\left(B_{j-a}\right)=B_{j}$. Applying the above comment to $i=j+a$ we find that $\sigma\left(B_{j+a}\right)=B_{j}$ and $\sigma\left(B_{j}\right)=B_{j+a}$. Therefore $j-a=j+a$, implying $a=n / 2$. This proves (i). 
Assume $L=4$. Every $\alpha_{\ell}(1 \leq \ell \leq n / 2-1)$ is clearly in $G_{\mathcal{A}}$ by Lemma 3.2 (i). Therefore $\left\langle\alpha_{0}, \alpha_{1}, \ldots, \alpha_{n / 2-1}\right\rangle \leq G_{\mathcal{A}}$. Pick $\sigma \in G_{\mathcal{A}}$. Since for every $i\left(i \in \mathbb{Z}_{n}\right)$ the automorphism $\sigma$ either fixes or interchanges $B_{i}$ and $B_{i+n / 2}$, we clearly have $G_{\mathcal{A}} \leq$ $\left\langle\alpha_{0}, \alpha_{1}, \ldots, \alpha_{n / 2-1}\right\rangle$. Therefore $G_{\mathcal{A}}=\left\langle\alpha_{0}, \alpha_{1}, \ldots, \alpha_{n / 2-1}\right\rangle$.

Assume $L$ is even, $L \neq 4$. By Lemma 3.2 (ii), $\left\langle\beta_{0}, \beta_{1}, \ldots, \beta_{N-1}\right\rangle \leq G_{\mathcal{A}}$. Pick $\sigma \in$ $G_{\mathcal{A}}$. For every $i\left(i \in \mathbb{Z}_{n}\right)$ the automorphism $\sigma$ either fixes or interchanges $B_{i}$ and $B_{i+n / 2}$. However, since $L \neq 4$, if $\sigma$ interchanges $B_{i}$ and $B_{i+n / 2}$, then it must interchange every pair of antipodal vertices of the inner cycle containing $B_{i}$ (and therefore also $B_{i+n / 2}$ ). Hence $G_{\mathcal{A}} \leq\left\langle\beta_{0}, \beta_{1}, \ldots, \beta_{N-1}\right\rangle$, implying $G_{\mathcal{A}}=\left\langle\beta_{0}, \beta_{1}, \ldots, \beta_{N-1}\right\rangle$.

Assume $L$ is odd. Again, by Lemma 3.2 (iii), we have $\left\langle\gamma_{0}, \gamma_{1}, \ldots, \gamma_{N / 2-1}\right\rangle \leq G_{\mathcal{A}}$. Pick $\sigma \in G_{\mathcal{A}}$ and assume that $\sigma$ interchanges $B_{i}$ and $B_{i+n / 2}$. Note that $B_{i}$ and $B_{i+n / 2}$ are now in different inner cycles. Therefore, $\sigma$ must interchange every $B_{j}$ of the inner cycle containing $B_{i}$ with $B_{j+n / 2}$ (which is contained in the same inner cycle as $B_{i+n / 2}$ ). It is now clear that $\sigma \in\left\langle\gamma_{0}, \gamma_{1}, \ldots, \gamma_{N / 2-1}\right\rangle$. This implies $G_{\mathcal{A}}=\left\langle\gamma_{0}, \gamma_{1}, \ldots, \gamma_{N / 2-1}\right\rangle$.

Proposition 3.4. Let $G_{\{\mathcal{A}\}}$ be the set-wise stabiliser of $\left\{A_{0}, A_{1}, \ldots, A_{n-1}\right\}$ in $G$. Then the following hold.

(i) If $a \neq n / 2$ then $G_{\{\mathcal{A}\}}=\langle\rho, \mu\rangle$.

(ii) If $a=n / 2$ and $L=4$, then $G_{\{\mathcal{A}\}}=\left\langle\rho, \mu, \alpha_{0}\right\rangle$.

(iii) If $a=n / 2, L$ is even and $L \neq 4$, then $G_{\{\mathcal{A}\}}=\left\langle\rho, \mu, \beta_{0}\right\rangle$.

(iv) If $a=n / 2$ and $L$ is odd, then $G_{\{\mathcal{A}\}}=\left\langle\rho, \mu, \gamma_{0}\right\rangle$.

Proof. Let $\sigma \in G_{\{\mathcal{A}\}}$. Observe that the group induced by $G_{\{\mathcal{A}\}}$ on $\mathcal{A}$ is $\langle\rho, \mu\rangle$, since the subgraph induced by $\mathcal{A}$ is a cycle. Therefore, $\rho^{k} \mu^{\ell} \sigma \in G_{\mathcal{A}}$ for appropriate $k \in \mathbb{Z}_{n}, \ell \in \mathbb{Z}_{2}$. The result now follows from Lemma 3.3 and Lemma 3.1.

Corollary 3.5. Assume the automorphism group of $R_{n}(a, r)$ has three orbits on the edgeset of $R_{n}(a, r)$ (that is, $R_{n}(a, r)$ does not satisfy any of the conditions ( $i$ ) and (ii) of Theorem 2.3). Then the following hold.

(i) If $a \neq n / 2$ then $G=\langle\rho, \mu\rangle$.

(ii) If $a=n / 2$ and $L=4$, then $G=\left\langle\rho, \mu, \alpha_{0}\right\rangle$.

(iii) If $a=n / 2, L$ is even and $L \neq 4$, then $G=\left\langle\rho, \mu, \beta_{0}\right\rangle$.

(iv) If $a=n / 2$ and $L$ is odd, then $G=\left\langle\rho, \mu, \gamma_{0}\right\rangle$.

Proof. If $R_{n}(a, r)$ has three orbits on the edge-set, then one of these three orbits is the set of rim edges. Therefore $G=G_{\{\mathcal{A}\}}$. The result now follows from Proposition 3.4.

We now turn our attention to the case when $R_{n}(a, r)$ has two orbits on edges. In this case, in view of Lemma 2.1, the rim edges and the hub edges are in the same orbit, implying that $\operatorname{gcd}(n, r)=1$. Additionally, every automorphism of such a rose window graph must either fix the rim and hub or interchange them. Now suppose that we have an automorphism $\omega$ that interchanges the rim and hub. For any automorphism $\delta$ of $R_{n}(a, r)$, we then have that $\omega \delta$ or $\delta$ is contained in $G_{\mathcal{A}}$ (noting that the set-wise stabilizer of $\left\{A_{i}: i \in \mathbb{Z}_{n}\right\}$ is the same as the set-wise stabilizer of $\left\{B_{i}: i \in \mathbb{Z}_{n}\right\}$ ). Thus in order to calculate the automorphism groups of such graphs, we need only find one $\omega$ that interchanges the rim 
and hub, and then $G=\left\langle G_{\mathcal{A}}, \omega\right\rangle$. Of course, $G_{\mathcal{A}}$ is given in Lemma 3.3, and we need only consider the parameters listed in Theorem 2.3.

Definition 3.6. (i) Assume $r^{2} \equiv \pm 1(\bmod n)$ and $r a \equiv-a(\bmod n)$. Then we define $\delta: V \rightarrow V$ by $\delta\left(A_{i}\right)=B_{r i}$ and $\delta\left(B_{i}\right)=A_{r i}$.

(ii) Assume $n$ is divisible by $4, r$ is odd, $a=n / 2$ and $\left(r^{2}+n / 2\right) \equiv \pm 1(\bmod n)$. Then we define $\gamma: V \rightarrow V$ by $\gamma\left(A_{i}\right)=B_{r i}$ and $\gamma\left(B_{i}\right)=A_{r i+(n / 2) i}$.

Lemma 3.7. Assume $r^{2} \equiv \pm 1(\bmod n)$ and $r a \equiv-a(\bmod n)$. Then $\delta \in G$, where $\delta$ is as defined in Definition 3.6(i).

Proof. Note that $\delta$ is a bijection since $\operatorname{gcd}(n, r)=1$. The proof of the fact that $\delta$ is an automorphism of $R_{n}(a, r)$ is straightforward.

Lemma 3.8. Assume $n$ is divisible by $4, r$ is odd, $a=n / 2$ and $\left(r^{2}+n / 2\right) \equiv \pm 1(\bmod n)$. Then $\gamma \in G$, where $\gamma$ is as defined in Definition 3.6(ii).

Proof. We will show that $\gamma$ is a bijection as once this is established it is straightforward to verify that $\gamma \in G$. Clearly, $\gamma$ maps $\left\{A_{i} \mid i \in \mathbb{Z}_{n}\right\}$ bijectively to $\left\{B_{i} \mid i \in \mathbb{Z}_{n}\right\}$ as $r$ is a unit. Similarly, $\gamma$ maps $\left\{B_{i} \mid i \in \mathbb{Z}_{n}, i\right.$ odd $\}$ bijectively to $\left\{A_{i}: i \in \mathbb{Z}_{n}, i\right.$ odd $\}$, and $\left\{B_{i} \mid i \in \mathbb{Z}_{n}, i\right.$ even $\}$ to $\left\{A_{i}: i \in \mathbb{Z}_{n}, i\right.$ even $\}$. Hence $\gamma$ is a bijection.

Corollary 3.9. Assume the automorphism group of $R_{n}(a, r)$ has two orbits on the edge-set of $R_{n}(a, r)$. Then, in view of Theorem 2.3, the following hold.

(i) If $a \neq n / 2$ and $r^{2} \equiv 1(\bmod n)$, then $G=\langle\rho, \mu, \delta\rangle$.

(ii) If $a=n / 2, r^{2} \equiv \pm 1(\bmod n)$ and $r a \equiv-a(\bmod n)$, then $G=\left\langle\rho, \mu, \beta_{0}, \delta\right\rangle$.

(iii) If $n$ is divisible by $4, r$ is odd, $a=n / 2$ and $\left(r^{2}+n / 2\right) \equiv \pm 1(\bmod n)$, then $G=\left\langle\rho, \mu, \beta_{0}, \gamma\right\rangle$.

We remark that in the case (ii) of the previous corollary when $r^{2}=-1$, listing $\beta_{0}$ as a generator is redundant as $\delta^{2} \mu=\beta_{0}$. In (iii), $\beta_{0}$ is superfluous as if $r^{2}+n / 2 \equiv-1(\bmod n)$ then $\beta_{0}=\gamma^{2} \mu$ while if $r^{2}+n / 2 \equiv 1(\bmod n)$ then $\beta_{0}=\rho^{-1} \gamma \rho^{r} \gamma$.

The full automorphism group of all rose window graphs are now known with the previous result. We may then check each case to determine which are vertex-transitive. But given that $\rho$ is always an automorphism of $R_{n}(a, r), R_{n}(a, r)$ is vertex-transitive if and only if there is an automorphism of $R_{n}(a, r)$ which maps a rim vertex to a hub vertex and an automorphism which maps a hub vertex to a rim vertex. Recall that a rose window graph has either three, two or one edge orbit. It has at most two edge orbits if and only if there is an automorphism which maps rim edges (vertices) to hub edges (vertices) and vice versa. Therefore, a rose window graph is vertex-transitive if and only if it has either one or two edge orbits. The edge-transitive rose window graphs are given in [5] and their full automorphism groups were obtained in [6]. Combining this information with Theorem 2.3, we obtain the following result which characterizes exactly which rose window graphs are vertex-transitive.

Theorem 3.10. Let $n \geq 3$ be an integer and $a, r \in \mathbb{Z}_{n} \backslash\{0\}$. The rose window graph $R_{n}(a, r)$ is vertex-transitive if and only if one of the following holds:

(i) $r^{2} \equiv \pm 1(\bmod n)$ and $r a \equiv \pm a(\bmod n)$; 
(ii) $n$ is divisible by $4, r$ is odd, $a=n / 2$ and $\left(r^{2}+n / 2\right) \equiv \pm 1(\bmod n)$;

(iii) $n$ is divisible by 2 , $a=n / 2 \pm 2$, and $r=n / 2 \pm 1$;

(iv) $n$ is divisible by $12, a= \pm(n / 4+2)$, and $r= \pm(n / 4-1)$ or $a= \pm(n / 4-2)$ and $r= \pm(n / 4+1) ;$ or

(v) $n$ is divisible by $2, a=2 b$, where $b^{2} \equiv \pm 1(\bmod n / 2)$, and $r$ is odd such that $r \equiv \pm 1(\bmod n / 2)$.

\section{Isomorphisms of non edge-transitive rose window graphs}

Let $R_{n}(a, r)$ and $R_{n}\left(a_{1}, r_{1}\right)$ be non edge-transitive rose window graphs. In this section we consider the problem of finding conditions on $a, r, a_{1}, r_{1}$ to ensure that $R_{n}(a, r)$ and $R_{n}\left(a_{1}, r_{1}\right)$ are isomorphic. For the remainder of this paper, we will, as usual, denote the vertices of $R_{n}(a, r)$ by $\left\{A_{0}, A_{1}, \ldots, A_{n-1}\right\} \cup\left\{B_{0}, B_{1}, \ldots, B_{n-1}\right\}$. The vertices of the rose window graph $R_{n}\left(a_{1}, r_{1}\right)$ will be denoted in the natural way by $\left\{C_{0}, C_{1}, \ldots, C_{n-1}\right\} \cup$ $\left\{D_{0}, D_{1}, \ldots, D_{n-1}\right\}$. Let $\rho$ and $\mu$ denote the automorphisms of $R_{n}(a, r)$ defined at the beginning of this paper, and $\rho_{1}$ and $\mu_{1}$ the corresponding automorphisms of $R_{n}\left(a_{1}, r_{1}\right)$.

Theorem 4.1. Let $R_{n}(a, r)$ and $R_{n}\left(a_{1}, r_{1}\right)$ be rose window graphs. If one of the following holds, then $R_{n}(a, r)$ and $R_{n}\left(a_{1}, r_{1}\right)$ are isomorphic:

(i) $r_{1}= \pm r$ and $a_{1}= \pm a$;

(ii) $\operatorname{gcd}(n, r)=1, r_{1}= \pm r^{-1}$, and $a_{1}= \pm a r^{-1}$;

(iii) $n$ is even with $\operatorname{gcd}(n, r)=\operatorname{gcd}(n, n / 2+r), a=a_{1}=n / 2$ and $r_{1}= \pm(r+n / 2)$;

(iv) $n$ is even with $\operatorname{gcd}(n, r)=\operatorname{gcd}(n, n / 2+r)=1, a=a_{1}=n / 2$ and $r_{1}= \pm(r+$ $n / 2)^{-1}$

(v) $r= \pm 1, r_{1}= \pm 1, \operatorname{gcd}(n, a)=\operatorname{gcd}\left(n, a_{1}\right)=2$ and $a a_{1} / 2 \equiv \pm 2(\bmod n)$;

(vi) $\operatorname{gcd}(n, n / 2-1)=1, r= \pm(n / 2-1), r_{1}= \pm(n / 2-1), \operatorname{gcd}(n, a)=\operatorname{gcd}\left(n, a_{1}\right)=2$ and $a a_{1} / 2 \equiv \pm 2(\bmod n)$.

Proof. (i) Note that $R_{n}(a, r)=R_{n}(a,-r)$ and that an isomorphism between $R_{n}(a, r)$ and $R_{n}(-a, r)$ is given by $\phi\left(A_{i}\right)=C_{-i}$ and $\phi\left(B_{i}\right)=D_{-i}$ for $i \in \mathbb{Z}_{n}$.

(ii) Assume $\operatorname{gcd}(n, r)=1, r_{1}=r^{-1}$, and $a_{1}=a r^{-1}$. Then an isomorphism from $R_{n}(a, r)$ to $R_{n}\left(a_{1}, r_{1}\right)$ is given by $\phi\left(A_{i}\right)=D_{-i r^{-1}}$ and $\phi\left(B_{i}\right)=C_{-i r^{-1}}$ for $i \in \mathbb{Z}_{n}$. The result now follows from (i) above.

(iii) Let $L=\frac{n}{\operatorname{gcd}(n, r)}=\frac{n}{\operatorname{gcd}(n, n / 2+r)}$, the length of the inner cycles of $R_{n}(a, r)$ and $R_{n}\left(a_{1}, r_{1}\right)$. We first claim that $L$ is divisible by 4 . To this end let $n=2^{i} n_{o}$ and $r=2^{j} r_{o}$, where $n_{o}$ and $r_{o}$ are odd positive integers. Since $\operatorname{gcd}(n, r)=\operatorname{gcd}(n, n / 2+r)$, we also have that $\operatorname{gcd}(n, r)=\operatorname{gcd}(n / 2, r)$, and so $j \leq i-1$. Assume now that $j=i-1$. Then $n / 2+r=2^{i-1}\left(n_{o}+r_{o}\right)=2^{i}\left(n_{o}+r_{o}\right) / 2$ (note that $n_{o}+r_{o}$ is even). This shows that $\operatorname{gcd}(n, n / 2+r)$ is divisible by $2^{i}$. Since $\operatorname{gcd}(n, r)$ is not divisible by $2^{i}$, we have a contradiction. Therefore, $j \leq i-2$, and so $L$ is divisible by 4 .

Now define $\phi: V\left(R_{n}(n / 2, r)\right) \mapsto V\left(R_{n}(n / 2, n / 2+r)\right)$ by $\phi\left(A_{i}\right)=C_{i}$ for $i \in \mathbb{Z}_{n}$ and $\phi\left(B_{\ell+k r}\right)=D_{\ell+k r+k n / 2}$ for $0 \leq \ell \leq \operatorname{gcd}(n, r)-1$ and $0 \leq k \leq L-1$. Choose an inner cycle $C$ of $R_{n}(n / 2, r)$. Note that $\phi$ maps $C$ to an inner cycle of $R_{n}(n / 2, n / 2+r)$, and while doing so, the only change in every other vertex is changing $B_{i}$ to $D_{i}$ and on the remaining vertices $\phi$ interchanges "antipodal vertices" of the cycle. This will produce a 
bijection if and only if $L$ is divisible by 4 , and so $\phi$ is a bijection. It is now routine to check that $\phi$ is an isomorphism. The result now follows from (i) above.

(iv) Immediately from (ii) and (iii) above.

(v) Assume $r=1, r_{1}=1, \operatorname{gcd}(n, a)=\operatorname{gcd}\left(n, a_{1}\right)=2$ and $a a_{1} / 2 \equiv 2(\bmod n)$. Define a mapping from $V\left(R_{n}(a, r)\right)$ to $V\left(R_{n}\left(a_{1}, r_{1}\right)\right)$ by $\phi\left(A_{2 i}\right)=C_{i a_{1}}, \phi\left(A_{2 i+1}\right)=$ $D_{i a_{1}}, \phi\left(B_{2 i}\right)=C_{i a_{1}+1}, \phi\left(B_{2 i+1}\right)=D_{i a_{1}+1}$ for $0 \leq i \leq n / 2-1$. Observe that $\phi$ is a bijection as $\operatorname{gcd}\left(n, a_{1}\right)=2$. It is also clear that $\phi$ is an isomorphism. The result now follows from (i) above.

(vi) Assume $r=n / 2-1, r_{1}=n / 2-1, \operatorname{gcd}(n, a)=\operatorname{gcd}\left(n, a_{1}\right)=2$ and $a a_{1} / 2 \equiv$ $2(\bmod n)$. Note that since $\operatorname{gcd}(n, n / 2-1)=1$, we have that $n / 2$ is even. Furthermore, since $\operatorname{gcd}(n, a)=\operatorname{gcd}\left(n, a_{1}\right)=2, a / 2$ and $a_{1} / 2$ are odd. Define a mapping from $V\left(R_{n}(a, r)\right)$ to $V\left(R_{n}\left(a_{1}, r_{1}\right)\right)$ by $\phi\left(A_{2 i}\right)=C_{i a_{1}}, \phi\left(A_{2 i+1}\right)=D_{i a_{1}}, \phi\left(B_{2 i}\right)=$ $C_{1+i a_{1}}, \phi\left(B_{2 i+n / 2+1}\right)=D_{1+i a_{1}}$ for $0 \leq i \leq n / 2-1$. Observe that $\phi$ is a bijection as $\operatorname{gcd}\left(n, a_{1}\right)=2$. Furthermore, since $a_{1} / 2$ is odd (and so $\left.(n / 4) a_{1}=n / 2\right), \phi$ is an isomorphism. The result now follows from (i) above.

Theorem 4.2. Let $\phi: R_{n}(a, r) \rightarrow R_{n}\left(a_{1}, r_{1}\right)$ be an isomorphism which sends every rim edge of $R_{n}(a, r)$ to a rim edge of $R_{n}\left(a_{1}, r_{1}\right)$. Then one of the following holds:

(i) $a_{1}= \pm a$ and $r_{1}= \pm r$;

(ii) $n$ is even with $\operatorname{gcd}(n, r)=\operatorname{gcd}(n, r+n / 2), a=a_{1}=n / 2$ and $r_{1}= \pm(r+n / 2)$.

Proof. Note that there exist $k \in \mathbb{Z}_{n}$ and $\ell \in\{0,1\}$ such that $\mu_{1}^{\ell} \rho_{1}^{k} \phi$ maps vertex $A_{i}$ to vertex $C_{i}$ for each $i \in \mathbb{Z}_{n}$. Therefore without loss of generality we can assume that $\phi$ maps vertex $A_{i}$ to vertex $C_{i}$ for each $i \in \mathbb{Z}_{n}$. Observe also that $\phi$ maps the hub (spoke) edges of $R_{n}(a, r)$ to the hub (spoke) edges of $R_{n}\left(a_{1}, r_{1}\right)$. It follows that $\phi\left(B_{0}\right) \in\left\{D_{0}, D_{-a_{1}}\right\}$.

Claim 1: If $\phi\left(B_{0}\right)=D_{0}$ then $a_{1}=a$. If, in addition, $a \neq n / 2$, then $r_{1}= \pm r$. Assume $\phi\left(B_{0}\right)=D_{0}$. Since vertices $B_{0}$ and $A_{a}$ are adjacent, vertices $\phi\left(B_{0}\right)=D_{0}$ and $\phi\left(A_{a}\right)=C_{a}$ are also adjacent. Since $a \neq 0$ this shows that $a_{1}=a$. Assume $a \neq n / 2$. As $A_{r}$ and $B_{r}$ are adjacent, $\phi\left(A_{r}\right)=C_{r}$ and $\phi\left(B_{r}\right)$ are also adjacent. This shows that $\phi\left(B_{r}\right) \in\left\{D_{r}, D_{r-a}\right\}$. As $A_{r+a}$ and $B_{r}$ are adjacent, $\phi\left(A_{r+a}\right)=C_{r+a}$ and $\phi\left(B_{r}\right)$ are also adjacent. This shows that $\phi\left(B_{r}\right) \in\left\{D_{r}, D_{r+a}\right\}$. Note that since $a \notin\{0, n / 2\}$, we have $\left\{D_{r}, D_{r-a}\right\} \cap\left\{D_{r}, D_{r+a}\right\}=\left\{D_{r}\right\}$. Therefore $\phi\left(B_{r}\right)=D_{r}$ and $r_{1}= \pm r$. This proves Claim 1.

Claim 2: If $\phi\left(B_{0}\right)=D_{-a_{1}}$ then $a_{1}=-a$. If, in addition, $a \neq n / 2$, then $r_{1}= \pm r$. Rearranging the subscripts of the vertices $\left\{D_{0}, D_{1}, \ldots, D_{n-1}\right\}$ according to the rule $x \rightarrow$ $x+a_{1}$ we get the graph $R_{n}\left(-a_{1}, r_{1}\right)$ instead of the graph $R_{n}\left(a_{1}, r_{1}\right)$. Furthermore, $\phi$ : $R_{n}(a, r) \rightarrow R_{n}\left(-a_{1}, r_{1}\right)$ now maps vertex $B_{0}$ to (the new) vertex $D_{0}$. By Claim 1 we have $-a_{1}=a$ and, if $a \neq n / 2, r_{1}= \pm r$. This proves Claim 2 .

Assume now $a=a_{1}=n / 2$ and $r_{1} \neq \pm r$. Similarly as above, we find $\phi\left(B_{0}\right) \in$ $\left\{D_{0}, D_{n / 2}\right\}$. If $\phi\left(B_{0}\right)=D_{0}$, then $\phi\left(B_{r}\right) \in\left\{D_{r_{1}}, D_{-r_{1}}\right\} \cap\left\{D_{r}, D_{r+n / 2}\right\}$. Since $r_{1} \neq$ $\pm r$, we have $r_{1}= \pm(r+n / 2)$. It is clear that we have $\operatorname{gcd}(n, r)=\operatorname{gcd}\left(n, r_{1}\right)=\operatorname{gcd}(n, r+$ $n / 2)$. The case $\phi\left(B_{0}\right)=D_{n / 2}$ is treated similarly.

Theorem 4.3. Let $\phi: R_{n}(a, r) \rightarrow R_{n}\left(a_{1}, r_{1}\right)$ be an isomorphism which sends every rim edge of $R_{n}(a, r)$ to a hub edge of $R_{n}\left(a_{1}, r_{1}\right)$. Then one of the following holds:

(i) $a_{1}= \pm a r^{-1}$ and $r_{1}= \pm r^{-1}$; 
(ii) $n$ is even with $\operatorname{gcd}(n, r)=\operatorname{gcd}(n, r+n / 2)=1, a=a_{1}=n / 2$ and $r_{1}= \pm(r+$ $n / 2)^{-1}$.

Proof. Since $\phi$ sends the rim edges of $R_{n}(a, r)$ to the hub edges of $R_{n}\left(a_{1}, r_{1}\right)$, it also sends the hub edges of $R_{n}(a, r)$ to the rim edges of $R_{n}\left(a_{1}, r_{1}\right)$. This shows that $\operatorname{gcd}(n, r)=$ $\operatorname{gcd}\left(n, r_{1}\right)=1$. Rearranging the vertices of $R_{n}\left(a_{1}, r_{1}\right)$ according to the rule $C_{i} \rightarrow D_{i r_{1}^{-1}}$ and $D_{i} \rightarrow C_{i r_{1}^{-1}}$ for $i \in \mathbb{Z}_{n}$ we obtain the graph $R_{n}\left(-a_{1} r_{1}^{-1}, r_{1}^{-1}\right)$ instead of the graph $R_{n}\left(a_{1}, r_{1}\right)$. Moreover, $\phi$ now satisfies the assumptions of Theorem 4.2. If Theorem 4.2 (i) holds, then $r_{1}= \pm r^{-1}$ and $a_{1}= \pm a r^{-1}$. If Theorem 4.2 (ii) holds, then $n$ is even with $\operatorname{gcd}(n, r+n / 2)=\operatorname{gcd}(n, r)=1, a=-a_{1} r_{1}^{-1}=n / 2$ and $r_{1}^{-1}= \pm(r+n / 2)$. Since $r_{1}$ is odd (recall that $\left.\operatorname{gcd}\left(n, r_{1}\right)=1\right),-a_{1} r_{1}^{-1}=n / 2$ is equivalent to $a_{1}=n / 2$. The result follows.

Theorem 4.4. Let $\phi: R_{n}(a, r) \rightarrow R_{n}\left(a_{1}, r_{1}\right)$ be an isomorphism which sends every rim edge of $R_{n}(a, r)$ to a spoke edge of $R_{n}\left(a_{1}, r_{1}\right)$. Then one of the following holds:

(i) $r= \pm 1, r_{1}= \pm 1, \operatorname{gcd}(n, a)=\operatorname{gcd}\left(n, a_{1}\right)=2$ and $a a_{1} / 2 \equiv \pm 2(\bmod n)$;

(ii) $\operatorname{gcd}(n, n / 2-1)=1, r= \pm(n / 2-1), r_{1}= \pm(n / 2-1), \operatorname{gcd}(n, a)=\operatorname{gcd}\left(n, a_{1}\right)=2$ and $a a_{1} / 2 \equiv \pm 2(\bmod n)$.

Proof. Observe first that as the rim edges of $R_{n}(a, r)$ are mapped to the spoke edges of $R_{n}\left(a_{1}, r_{1}\right)$, the outer cycle of $R_{n}(a, r)$ is mapped to a cycle of even length in $R_{n}\left(a_{1}, r_{1}\right)$. This shows that $n$ is even. Next, as the rim edges of $R_{n}(a, r)$ are mapped to the spoke edges of $R_{n}\left(a_{1}, r_{1}\right)$, the image of a rim edge has endpoints a rim vertex and a hub vertex. As hub edges and rim edges have no endpoints in common, the images of hub edges and rim edges have no endpoints in common. This implies that hub edges cannot be mapped either to the hub edges or the rim edges, and so the hub edges of $R_{n}(a, r)$ are also mapped to the spoke edges of $R_{n}\left(a_{1}, r_{1}\right)$. As the spoke edges of $R_{n}\left(a_{1}, r_{1}\right)$ form a single edge orbit, we have that the hub and rim edges of $R_{n}(a, r)$ also forms a single edge orbit. This shows that $R_{n}(a, r)$ and $R_{n}\left(a_{1}, r_{1}\right)$ have two orbits on edges (and so $\operatorname{gcd}(n, r)=\operatorname{gcd}\left(n, r_{1}\right)=1$ ). We may thus assume that $\phi\left(A_{0}\right)=C_{i}$ and $\phi\left(A_{1}\right) \in\left\{D_{i}, D_{i-a_{1}}\right\}$ for some $i \in \mathbb{Z}_{n}$. Multiplying $\phi$ by appropriate powers of $\mu_{1}$ and $\rho_{1}$ we can further assume that $\phi\left(A_{0}\right)=C_{0}$ and $\phi\left(A_{1}\right)=D_{0}$. This implies that $\phi\left(A_{2 i}\right)=C_{i a_{1}}$ and $\phi\left(A_{2 i+1}\right)=D_{i a_{1}}$ for $0 \leq i \leq$ $n / 2-1$. Therefore, the order of $a_{1}$ in $\mathbb{Z}_{n}$ is $n / 2$ and thus $\operatorname{gcd}\left(n, a_{1}\right)=2$. Reversing the role of $R_{n}(a, r)$ and $R_{n}\left(a_{1}, r_{1}\right)$ we also obtain that $\operatorname{gcd}(n, a)=2$. Note that this also shows that $n, a$ and $a_{1}$ are all even. Since $R_{4}(2,1)$ is edge-transitive, we may assume that $n \geq 6$.

Observe now that $\phi\left(B_{0}\right) \in\left\{C_{1}, C_{-1}\right\}$. We will assume $\phi\left(B_{0}\right)=C_{1}$; the case $\phi\left(B_{0}\right)=C_{-1}$ is treated similarly. Since $B_{0}$ and $A_{a}$ are adjacent, $\phi\left(B_{0}\right)=C_{1}$ and $\phi\left(A_{a}\right)=C_{(a / 2) a_{1}}$ are also adjacent. This shows that $a a_{1} / 2 \equiv 2(\bmod n)$. Furthermore, $\phi\left(B_{2 i r}\right) \in\left\{C_{0}, C_{1}, \ldots, C_{n-1}\right\}$ and $\phi\left(B_{(2 i+1) r}\right) \in\left\{D_{0}, D_{1}, \ldots, D_{n-1}\right\}$ for $0 \leq i \leq$ $n / 2-1$.

Recall that $\operatorname{gcd}(n, r)=\operatorname{gcd}\left(n, r_{1}\right)=1$. In particular, $r, r_{1}, r^{-1}$ and $r_{1}^{-1}$ are odd. Since $B_{1}=B_{r^{-1} r}$ this shows that $\phi\left(B_{1}\right) \in\left\{D_{0}, D_{1}, \ldots, D_{n-1}\right\}$. Since $B_{1}$ and $A_{1}$ are adjacent, $\phi\left(B_{1}\right)$ and $\phi\left(A_{1}\right)=D_{0}$ are also adjacent. Since $B_{1}$ and $A_{a+1}$ are adjacent, $\phi\left(B_{1}\right)$ and $\phi\left(A_{a+1}\right)=D_{(a / 2) a_{1}}=D_{2}$ are also adjacent. Therefore $\phi\left(B_{1}\right) \in$ $\left\{D_{r_{1}}, D_{-r_{1}}\right\} \cap\left\{D_{2+r_{1}}, D_{2-r_{1}}\right\}$. This shows that $r_{1}= \pm 1$ or $r_{1}= \pm(n / 2-1)$. Reversing the role of $R_{n}(a, r)$ and $R_{n}\left(a_{1}, r_{1}\right)$ we also obtain that $r= \pm 1$ or $r= \pm(n / 2-1)$. 
Since $R_{n}(a, r)=R_{n}(a,-r)$, we need only show $r=1$ and $r_{1}=n / 2-1$ or $r=n / 2-1$ and $r_{1}=1$ cannot occur.

Suppose that $r=1$ and $r_{1}=n / 2-1$. We saw in the previous paragraph that $\phi\left(B_{1}\right) \in$ $\left\{D_{r_{1}}, D_{-r_{1}}\right\} \cap\left\{D_{2+r_{1}}, D_{2-r_{1}}\right\}$. Since $n \geq 6$ this implies $\phi\left(B_{1}\right)=D_{n / 2+1}$. But $B_{0}$ and $B_{1}$ are adjacent, and so $\phi\left(B_{0}\right)=C_{1}$ and $\phi\left(B_{1}\right)=D_{n / 2+1}$ are also adjacent. As $1 \neq n / 2+1$ this implies $n / 2+1+a_{1}=1$ and thus $a_{1}=n / 2$. It follows that $\operatorname{gcd}\left(n, a_{1}\right)=$ $n / 2 \geq 3$, contradicting $\operatorname{gcd}\left(n, a_{1}\right)=2$.

Finally, if $r=n / 2-1$ and $r_{1}=1$, then by reversing the roles of $R_{n}(a, r)$ and $R_{n}\left(a_{1}, r_{1}\right)$, this case cannot occur by arguments in the previous case.

\section{References}

[1] M. Boben, T. Pisanski and A. Žitnik, I-graphs and the corresponding configurations, J. Combin. Des. 13 (2005), 406-424.

[2] E. Dobson and D. Witte, Transitive permutation groups of prime-squared degree, J. Algebraic Combin. 16 (2002), 43-69.

[3] Y.-Q. Feng, J. H. Kwak, M.-Y. Xu, and J.-X. Zhou, Tetravalent half-arc-transitive graphs of order $p^{4}$, European J. Combin. 29 (2008), 555-567.

[4] R. Frucht, J. E. Graver and M. E. Watkins, The groups of the generalized Petersen graphs, Proc. Cambridge Philos. Soc. 70 (1971), 211-218.

[5] I. Kovács, K. Kutnar and D. Marušič, Classification of edge-transitive rose window graphs, $J$. Graph Theory 65 (2010), 216-231.

[6] I. Kovács, K. Kutnar and J. Ruff, Rose window graphs underlying rotary maps, Discrete Math. 310 (2010), 1802-1811.

[7] D. Marušič and C. E. Praeger, Tetravalent graphs admitting half-transitive group actions: alternating cycles, J. Combin. Theory Ser. B 75 (1999), 188-205.

[8] J. D. P. Meldrum, Wreath products of groups and semigroups, Pitman Monographs and Surveys in Pure and Applied Mathematics, vol. 74, Longman, Harlow, 1995.

[9] M. Petkovšek and H. Zakrajšek, Enumeration of I-graphs: Burnside does it again, Ars Math. Contemp. 2 (2009), 241-262.

[10] P. Potočnik, A list of 4-valent 2-arc-transitive graphs and finite faithful amalgams of index (4, 2), European J. Combin. 5 (2009), 1323-1336.

[11] P. Potočnik and S. Wilson, Linking ring structures and tetravalent semisymetric graphs, Ars Math. Contemp. 7 (2014), 341-352.

[12] P. Potočnik, P. Spiga and G. Verret, A census of small tetravalent arc-transitive graphs, htt $\mathrm{p}$ : //www.fmf.uni-lj.si/ potocnik/work_datoteke/Census4val-640.mgm

[13] P. Potočnik, P. Spiga and G. Verret, Tetravalent arc-transitive graphs with unbounded vertexstabilizers, Bull. Aust. Math. Soc. 84 (2011), 79-89.

[14] P. Potočnik, P. Spiga and G. Verret, Cubic vertex-transitive graphs on up to 1280 vertices, $J$. Symb. Comp. 50 (2013), 465-477.

[15] G. Sabidussi, On a class of fixed-point-free graphs, Proc. Amer. Math. Soc. 9 (1958), 800-804.

[16] A. Steimle and W. Staton, The isomorphism classes of the generalized Petersen graphs, Discrete Math. 309 (2009), 231-237.

[17] X. Wang and Y.-Q. Feng, Tetravalent half-edge-transitive graphs and non-normal Cayley graphs, J. Graph Theory 70 (2012), 197-213. 
E. Dobson et al.: The automorphism groups of non-edge-transitive rose window graphs

[18] S. Wilson, Rose window graphs, Ars Math. Contemp. 1 (2008), 7-19. 